\section{Über SteinzeugkühIschlangen.} Von K. Grote.

Ein Artikel von Gustav $R$ a u ter in No. 45 v. J. der "Zeitschrift für angewandte Chemie" übev "Kühlschlangen aus Steinzeug" darf im Interesse aller Fachgenossen, die grösseren Bedarf an Thonschlangen zu Kühlzwecken haben, nicht ohne weitere Erörterung bleiben. Thonschlangen sind kein billiger Artikel, und in einigen chemischen Industrien ist der Bedarf an denselben so gross, dass der Fabrikant aufs sorgfältigste seine Auswahl zu treffen gezwungen ist. Unsere gesammte Thonwaarentechnik hat sich im Laufe des letzten Jahrzehnts sehr vervollkommnet und unbedenklich kann man behaupten, dass der Forderung Rauter's, dass nur bestes Rohmaterial bei gewissenhaftester Ausführung verwendet werde, vou allen unseren grösseren Firmen nuchgekommen wird. Betreffs der Kühlschlangen nun sind in den letzten Jahren noch zwei Neuerungen aufgekommen, von denen man sich grosse Vortheile versprach, die Fabrikation von Kühl schlangen mit gewelltem Querschnitt wie solche von lose gelagerten Kühlschlangen. Rauter vertritt in seinem Aufsatze die Ansicht, dass beide Neuerungen nicht vortheilhaft sind, und zwar hauptsächlich deshalb, weil sowohl die gewellten wie auch die lose gelagerten Schlangen sich nach einer Beschädigung weniger leicht repariren lassen, wie die gewöhnlichen glatten, mit den Stegen festverbundenen Schlangen. Wer aberKühlschlangen braucht, wird sich nicht fragen, welche sich am leichtesten repariren lassen, sondern welche am wenigsten leicht brechen und welche die grösste Leistungsfähigkeit haben. Und in dieser Beziehung gebe ich unbedingt den lose gelagerten Schlangen den Vorzug, indem ich glcichzeitig auch noch bestreite, dass dieselben schwerer zu repariren sind als die fest gelagerten. Theoretisch müssten ja die Schlangen mit gewelltem Querschnitt die grösste Leistungsfähigkeit bieten, da sie die grösste Kühlfläche bei gleicherRohrweitehaben. Diese Schlangen haben sich aber nicht immer bewährt, und ist man von ihrer Verwendung wieder abgekommen. Der Grund liegt aber nicht ao der von Rauter behaupteten Reparaturunfäbigkeit, sondern an der ungleichmässigen Kühlung. Die Rohre der Schlangen liegen so, dass entweder eine Spitze oder eine Kehle nach oben liegt. In beiden Fillen lagert sich die Abscheidung aus dem Kühlwasser, die stets an den heissen Schlangen stattfindet, in den oberen Kehlen ab und kann hier das Kühlwasser nicht mehr wirken, wodurch lokale Überhitzung und daher Sprung entsteht. Nur bei sehr reinem Wasser sollte man daher gewellte Schlangen benutzen.

Rauter wendet sich speciell gegen die lose gelagerten Schlangen, womit er jedenfalls die unter No. 75441 patentirten - ein anderes Patent für lose Schlangen existirt meines Wissens nicht treffen will. Er behauptet, dass diese Schlangen schon beim Transport und beim Einsetzen in den Kühlbottich leichter dem Zerbrechen unterworfen sind als die festen Schlangen. Ich habe viele Dutzende von losen Schlangen bezogen, und nicht eine einzige ist trotz recht weiten Transports gebrochen oder beschädigt angekommen. Im Gegentheil muss man annehmen, dass lose Rohre eher noch Stösse der Kisten and dergl. vertragen liönnen, weil bei einem solchen Stoss das lose Rohr etwas ausweichen kann, während das angarnirte Rohr demselben wohl oder übel Stand halten muss oder zerbricht. Ich habe nuzählige lose Schlangen in Kühlbottiche einsetzen Iassen, aber nicht eine einzige hat dabei Schaden gelitten. Im Gegentheil ist bei den losen Schlangen die Anbringung eines Flascheuzuges viel bequemer, da man die Haken desselben ohne Weiteres in das Traggerüst einhängen kann, was bei den festen Schlangen nicht der Fall ist.

Es dürfte schwer zu beweisen sein, dass die losen Schlangen einen so starken Auftrieb erleiden, dass die Gefahr des Bruches wächst. Es kann gewiss nur vortheilhaft sein, wenn die ganze Schlange sozusagen anf døm Kissen des Kühlwassers mitruht, während die eigentliche Traglast nach wie vor die Knacken ausüben; denn Rauter wird wohl nicht behaupten wollen, dass die Schlange auf dem Wasser schwimmt.

Auch die Behauptung des Herrn Collegen, dass die losen Schlangen sich schwerer repariren lassen als die festen, bedarf des Beweises. Bei den festen Schlangen erleidet das Rohr beim Angarniren am Steg stets einen Eingriff in den Scherben. Hier springt deshalb die Schlange am leichtesten. Dann ist aber das Flicken eben wegen des Steges selbst fast unmöglich. Die Iosen Schlangen lassen sich aber auf ihrer Unterlage verschieben, und so kann man stets die Sprungstelle nach einem Punkt zwischen zwei Knacken hinbringen, sie umwickeln und die Limwicklung im Nothfalle mit Zuhülfenahme der Knacken unterstützen, so dass dieselbe nicht, was $R$ a uter als so gefährlich hinstellt, mit tragen helfen muss.

Für die losen Kühlschlangen spricht aber vor allem ihre grössere Leistungsfähigkeit. Ich habe z. B. in der Salpetersäurefabrikation gleichzeitig mit festen und mit losen Schlangen gearbeitet, mit ersteren aber im Maximum nur 80, mit letzteren durchschnittlich über 200 Operationen machen können. Es ist unbestreitbar, dass die losen Schlangen gleichmässiger in den Scherben sind, da sie keine Verdickungen an den Angarnirungsstellen haben; es ist unbestreitbar, dass die Schlangen, lose wie feste, sich durch die Wärme der durchlanfenden Flüssigkeit etwas ausdehnen und dass, während die losen Schlangen sich frei bewegen und ausdehnen können, die festgaruirten eine starke Spaunung zwischen den Angarnirungsstellen aushalten müssen, die schliesslich zum Bruch führt.

Wenn Rauter sich auf ein Schreiben einer rheinischen Fabrik bezieht, so kann man darauf nur erwidern, dass die betreffende Firma offenbar nicht mit den Schlangen umzugehen versteht. Einen verhängnissvollen Fehler kann man allerdings dadurch begehen, dass man auf die oberste Schlangenwindung ein zu schweres Übergangsrohr als Verbindung mit dem Destillirapparat aufsetzt. Das sollte man in jedem Falle - auch bei den festen Schlangen-vermeiden and $z$ wischen Destillirblase und Kühler lieber poeh ein $\mathrm{Zw}$ ischentourill einsetzen, so dass man kürzere Übergangsrohre verwenden kann. A uch ist es empfehlenswerth, das Übergangsrohr nach dem Kühler in der Richtung der Schlangenwindung anzubringen, so dass die Gase ohne Stoss aus der Blase bez. dem Zwischengefäss durch die KühIschlange geleitet werden. 